\title{
Activated Platelets Harbor SARS-CoV-2 during Severe COVID-19
}

\author{
Ejaife O. Agbani ${ }^{1,2}$ Prism Schneider ${ }^{3,4}$ Braedon McDonald ${ }^{5}$ Leslie Skeith ${ }^{6}$ Man-Chiu Poon ${ }^{6,7}$ \\ Adrienne Lee $^{6}$
}

\footnotetext{
${ }^{1}$ Department of Physiology and Pharmacology, Cumming School of Medicine, University of Calgary, Alberta, Canada

2 Libin Cardiovascular Institute, University of Calgary, Alberta, Canada

3 Department of Surgery, Cumming School of Medicine, University of Calgary, Alberta, Canada

${ }^{4}$ McCaig Institute for Bone and Joint Health, University of Calgary, Alberta, Canada

${ }^{5}$ Snyder Institute for Chronic Diseases, Department of Critical Care Medicine, Cumming School of Medicine, University of Calgary, Alberta, Canada

${ }^{6}$ Division of Hematology and Hematological Malignancies,

Department of Medicine, Cumming School of Medicine, University of Calgary, Alberta, Canada

${ }^{7}$ Arnie Charbonneau Cancer Institute, University of Calgary, Alberta,
} Canada

Thromb Haemost 2022;122:308-309.

\section{Description}

Clinical SARS-CoV-2 (severe acute respiratory syndrome coronavirus 2) infection (coronavirus disease 2019 [COVID-19]) is characterized by a hyperinflammatory and procoagulant state that increases the risk of thrombosis and death. Despite thromboprophylaxis at conventional doses, incidence as high as $31 \%$ has been reported for thrombotic complications in intensive care unit (ICU) patients with COVID-19. ${ }^{1}$ We utilized a state-of-the-art high-resolution three-dimensional (3D) imaging approach to examine the interactions of SARS-CoV-2 with platelets, erythrocytes, and leucocytes in blood samples obtained from COVID-19 patients in our ICU. We conjugated primary antibodies to SARS-CoV-2 matrix (membrane), nucleoprotein, and the extracellular domain $\mathrm{S} 1+\mathrm{S} 2$ spike protein to Alexa-Fluor secondary antibodies. We then visualized platelet procoagulant activity and the spatial localization of SARS-CoV-2 in platelet-rich-plasma reconstituted to contain erythrocytes and leucocytes. The images in $\mathbf{- F i g . 1 A}$ are twodimensional projections of 3D data stacks (extended focus), and the main image in this - Fig. $\mathbf{~ A ~}$ is a superimposition of the images in the single-channel inserts. - Fig. 1A images show in blue activated platelets, morphologically transformed and expressing membrane P-selectin in a patient who succumbed to SARS-CoV-2 infection 5 days after this blood sample was

received

June 18, 2021

accepted after revision

October 29, 2021

published online

November 2, 2021
Address for correspondence Ejaife O. Agbani, BPharm, MSc, PhD, Department of Physiology and Pharmacology, Cumming School of Medicine, University of Calgary, Calgary, T2N 4N1 Alberta, Canada (e-mail: ejaife.agbani@ucalgary.ca).

collected. In - Fig. 1A, mouse monoclonal antibody against human Glycophorin-A was custom conjugated with AlexaFluor 647 and used to detect Glycophorin-A, the major sialoglycoprotein expressed on erythrocytes and erythroid precursor cells. P-selectin expression on activated platelets was detected by fluorescence signals of Alexa-Fluor 488 Antihuman CD62P antibody. Also, platelet membrane thrombin generation was detected using Alexa-Fluor-conjugated mouse monoclonal antibody specific for an epitope mapping between amino acids 331-376 within an internal region of human thrombin (data not shown). Here, the activated platelet, but not erythrocytes, is shown to have internalized SARS-CoV-2 (in red) into the cytosol ( $\mathbf{F i g}$. 1A, B), probably via a passive mechanism, as we have previously established that actin cytoskeleton remodeling and increased membrane permeability occurred during platelet transformation to the procoagulant phenotype. ${ }^{2,3}$ The white-arrowed platelet in - Fig. $\mathbf{1 A}$ is shown in - Fig. 1B in a 3D orientation ( - Fig. 1B-i) to highlight a location of SARS-Cov-2 in the platelet cytosol. The associated supplementary data ( - Video 1 ) show the spatial distribution of SARS-Cov-2 within the cytosol as the platelet is examined via $X Y Z, X Z, Y Z$, and $X Y$ planes. Selected images of $X Y$ and $X Z$ planes of the same platelet are shown in - Fig. 1B-ii and B-iii, respectively. We used a Nikon A1R laser scanning confocal microscope to capture images at Nyquist via Nikon NIS- (c) 2021. Thieme. All rights reserved. Georg Thieme Verlag KG,

Rüdigerstraße 14,

70469 Stuttgart, Germany
DOI https://doi.org/ 10.1055/a-1683-8455. ISSN 0340-6245. 

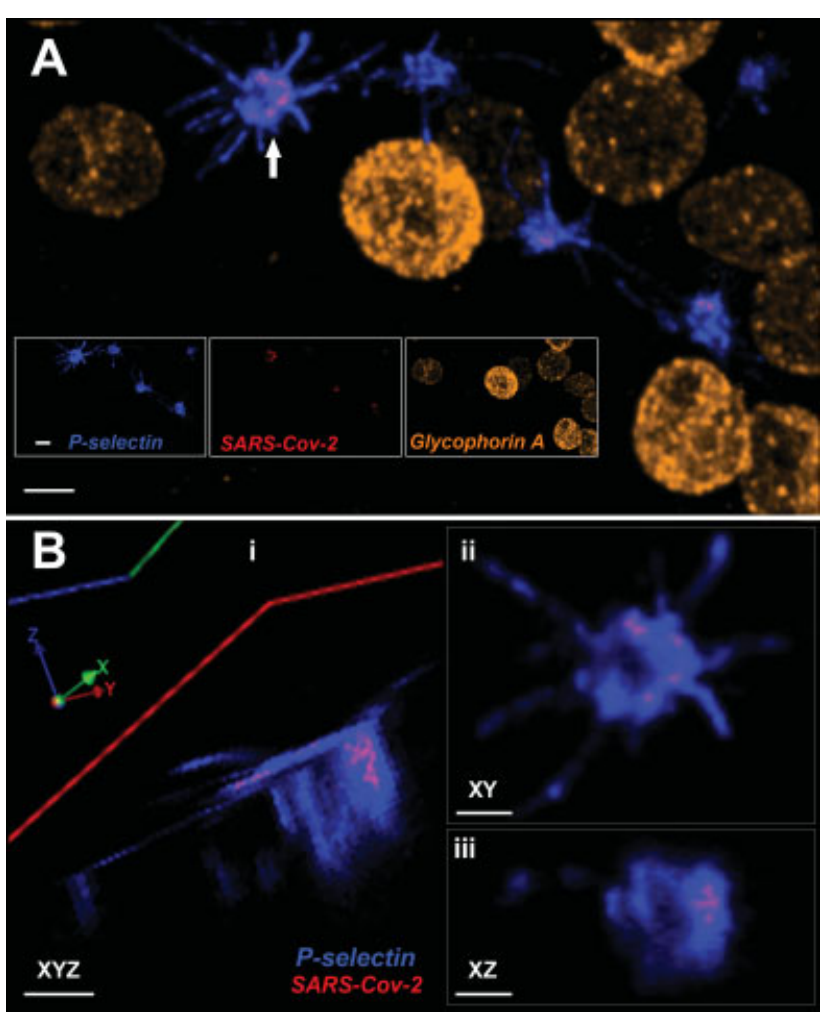

Fig. 1 Activated platelets harbor SARS-CoV-2 during severe COVID19.

Elements imaging software, and by means of an oil immersion Plan Apo Lambda objective lens (60x; numerical aperture: 1.4; working distance: $0.13 \mathrm{~mm}$ ). The acquisition involved fast and sensitive four-color confocal imaging and transmitted light.
The acquisition setting was kept constant, at high-speed/highdefinition resonant scanning (up to $1,024 \times 1,024$ pixels). Image resolution was improved by the restoration complement of Volocity imaging Software Suite, and analyzed using the same software (Quorum Technologies Inc., Canada). Scale bars: $3 \mu \mathrm{m}(\mathrm{A})$ and $2 \mu \mathrm{m}(\mathrm{B})$.

\section{Video 1}

Activated platelets harbor SARS-CoV-2 during severe COVID-19. Online content including video sequences viewable at: https://www.thieme-connect.com/ products/ejournals/html/10.1055/a-1683-8455.

\section{Funding}

This work was supported by the Live Cell Imaging Facility, funded by the Snyder Institute at the University of Calgary.

\section{Conflict of Interest}

None declared.

\section{References}

1 Klok FA, Kruip MJHA, van der Meer NJM, et al. Incidence of thrombotic complications in critically ill ICU patients with COVID-19. Thromb Res 2020;191:145-147

2 Agbani EO, Poole AW. Procoagulant platelets: generation, function, and therapeutic targeting in thrombosis. Blood 2017;130 (20):2171-2179

3 Agbani EO, van den Bosch MTJ, Brown E, et al. Coordinated membrane ballooning and procoagulant spreading in human platelets. Circulation 2015;132(15):1414-1424 